\title{
Nontraumatic Intracranial Hemorrhage
}

\author{
Pedro Vilela and Martin Wiesmann
}

\section{Learning Objectives}

- To understand typical imaging characteristics of intracranial hemorrhage in CT and MR imaging

- To know the most likely differential diagnosis of pathologies responsible for the hemorrhage with reference to anatomical location and patient characteristics

- To know how to triage patients suffering from an intracranial hemorrhage for further imaging and therapy

\subsection{Introduction}

Stroke is a major global health problem with different expressions among the globe but with a significant impact in the countries. The global prevalence of stroke varies from 339 to 1184 per 100,000, being higher among the more developed countries that face the problem of aging of the population. The prevalence of hemorrhagic strokes also varies among countries from 32-270 per 100,000 [1].

Spontaneous intracranial hemorrhage (IH) accounts for up to $10-15 \%$ of all strokes and is an important cause of neurological morbidity and mortality. The intracranial hemorrhage incidence is estimated in 10-30/100,000, increasing with age (doubles each decade after 35 years of age) [2].

The 1-year survival is only about $30 \%$, ranging the 30-day mortality between 35 and $52 \%$, with approximately half of the deaths occurring during the first 2 days after presentation [2]. The prognosis of IH mainly depends on the severity of intracra-

P. Vilela

Neuroradiology Department, Hospital Beatriz Angelo, Loures, Portugal

Neuroradiology Department, Hospital da Luz, Lisbon, Portugal

M. Wiesmann $(\bowtie)$

Department of Diagnostic and Interventional Neuroradiology, University Hospital, Technical University of Aachen RWTH,

Aachen, Germany

e-mail:mwiesmann@ukaachen.de nial hypertension and associated complications such as brain herniation, the development of hydrocephalus, and for subarachnoid hemorrhage on the development of arterial vasospasm.

Spontaneous intracranial hemorrhage may be parenchymal, intraventricular, subarachnoid, subdural, and epidural, the former being the most common location and the latter two being uncommon and usually associated with trauma.

Computed tomography $(\mathrm{CT})$ remains the standard method to detect intracranial hemorrhage although radiologists need also to be familiar with the appearance of hematomas using magnetic resonance imaging (MRI). In this paper the authors suggest a structured imaging diagnostic approach for spontaneous intracranial hemorrhage and review the imaging findings of the most common causes.

\subsection{Hemorrhage Imaging}

In the presence of a stroke with acute neurological deficit an emergent imaging study is warranted to evaluate the presence of ischemic or hemorrhagic stroke. Generally, CT is the initial imaging method used, due to its wider availability, rapid acquisition, lack of contraindications, and high accuracy to depict intracranial hemorrhage.

Imaging has a crucial role on intracranial hemorrhage evaluation. Firstly, it defines the type of stroke (ischemic or hemorrhagic), which have completely different managements. It is also essential to identify the anatomic compartment the lesion is located in, and approximate the age of the hemorrhage. The next step is to triage patients into those likely to have an underlying cause (Table 5.1) which requires urgent diagnosis and treatment, and those who do not require urgent interventions. To do this, neuroimaging findings need to be considered in combination with the age and medical history of the patient. In the setting of acute intracranial hemorrhage, CT angiography (or MR angiography) can be used to screen for the presence of vascular abnormalities such as intracranial aneurysmas, arteriovenous malformations, or 
Table 5.1 Most common etiologies of nontraumatic intracranial hemorrhage by age group and anatomic location

\begin{tabular}{|c|c|c|c|c|}
\hline & \multicolumn{2}{|l|}{ Adult } & \multicolumn{2}{|l|}{ Pediatric } \\
\hline & Elderly & Young/middle aged & Adolescent/child/infant & Newborn \\
\hline Parenchymal & $\begin{array}{l}\text { - Hypertensive hemorrhage } \\
\text { - Cerebral amyloid angiopathy (CAA) } \\
\text { - Hemorrhagic transformation of acute } \\
\text { ischemic infarction } \\
\text { - Dural AVF } \\
\text { - Venous thrombosis } \\
\text { - Coagulopathies } \\
\text { - Neoplasms }\end{array}$ & $\begin{array}{l}\text { - } \text { Brain AVM } \\
\text { - Cavernomas } \\
\text { - Venous thrombosis } \\
\text { - Hypertensive } \\
\text { encephalopathies/PRES } \\
\text { - RVCS } \\
\text { - Drug abuse } \\
\text { - Neoplasms }\end{array}$ & $\begin{array}{l}\text { - Brain AVM } \\
\text { - Cavernomas } \\
\text { - Venous thrombosis } \\
\text { - Tumors } \\
\text { - Coagulopathies } \\
\text { (congenital/acquired) }\end{array}$ & $\begin{array}{l}\text { - Hypoxic-ischemic } \\
\text { injury }\end{array}$ \\
\hline Subarachnoid & $\begin{array}{l}\text { - Aneurysm } \\
\text { - Cerebral amyloid angiopathy (CAA) } \\
\text { - Perimesencephalic hemorrhage } \\
\text { - Bleeding diathesis (e.g., excessive } \\
\text { anticoagulation) }\end{array}$ & $\begin{array}{l}\text { - Aneurysm } \\
\text { - Arterial dissection } \\
\text { - Brain AVM }\end{array}$ & \multicolumn{2}{|c|}{$\begin{array}{l}\text { - Aneurysm } \\
\text { - Sickle cell disease (in children) } \\
\text { - Coagulopathies (congenital/acquired) }\end{array}$} \\
\hline $\begin{array}{l}\text { Epidural/ } \\
\text { subdural }\end{array}$ & \multicolumn{2}{|c|}{$\begin{array}{l}\text { - Dural AVF } \\
\text { - Aneurysm } \\
\text { - Moyamoya syndrome } \\
\text { - Dural metastases } \\
\text { - Bleeding diathesis/hematological disorders }\end{array}$} & & \\
\hline
\end{tabular}

Table 5.2 CT and MR characteristics of intracranial hemorrhage

\begin{tabular}{|c|c|c|c|c|c|}
\hline Time & NECT & CECT & T1-weighted & T2-weighted & $\mathrm{T} 2 * \mathrm{~W}$ \\
\hline Hyperacute $(<6$ h) & Hyperdense & & Iso & Hyper & Hypo \\
\hline Acute (6 h-3 days) & Hyper & & Iso & Нypo & Нypo \\
\hline \multirow[t]{2}{*}{ Early subacute (3-7 days) } & \multirow{2}{*}{ Hyper/iso } & & Iso (center) & Нyро & Нypo \\
\hline & & & Hyper (rim) & & \\
\hline Late subacute (1-4 weeks) & Iso/hypo & Rim enhancement & Hyper & Hyper & Hypo \\
\hline Chronic (months-years) & Нyро & & Нуро & Нуро & Нyро \\
\hline
\end{tabular}

Density/signal intensity compared to cortex. NECT non-enhanced CT, CECT contrast enhanced CT

dural arteriovenous fistulas. Thus, imaging is essential to evaluate critical situations, to establish the prognosis, and to guide the treatment.

It is important to highlight that the blood imaging signs will vary over time in both computed tomography (CT) and magnetic resonance imaging (MRI), as shown in Table 5.2. Evolution over time will vary according to the location of the hemorrhage, with a faster clearance in the CSF spaces.

To understand the appearance of blood clots on CT and MRI, some knowledge on clot formation is helpful.

Primary acute intracerebral hemorrhages appear as lesions of homogenous hyperattenuation (Figs. 5.1 and 5.2) on (noncontrast enhanced) CT. The hyperattenuation results from the increasing protein density within the hematoma. As the hematoma matures, there is progressive clot formation and retraction with fluid loss, which then causes the hyperattenuation to increase from a range of 40-80 HU to $80-100 \mathrm{HU}$ [3]. In the acute phase, the presence of fluid-fluid levels and/or hypoattenuated areas inside the hematoma may represent a hyperacute hematoma, active bleeding (with the new incoming blood corresponding to these hypoattenuating areas), and/or the presence of coagulation disorder (acquired or congenital). The "swirl sign" characterized by the mixture of different densities or MR signal intensities in an acute hemorrhage suggest an active bleeding. Typically, clots become isodense to brain on CT images at 8-14 days after hemorrhage, then progressively hypodense after 2-4 weeks and, finally, at the late subacute and chronic phases, they are usually hypodense approaching the attenuation of cerebrospinal fluid (CSF).

For MRI it is also important to understand the type of hemoglobin present at each stage of the hematoma. This allows the estimation of the age of the hematoma. Initially, an intracerebral hematoma consists of intact red blood cells (RBCs) containing mainly oxygen-saturated hemoglobin (oxyhemoglobin, $\mathrm{OxyHb}$ ). The hemoglobin is then gradually deoxygenated. At $48 \mathrm{~h}$ after hemorrhage the clot consists almost entirely of deoxyhemoglobin (DeoxyHb). At this stage, the hematoma is surrounded by edema. In the early subacute phase (3-7 days) DeoxyHb is gradually converted to methemoglobin (MetHb). These changes begin at the periphery of the clot and then progress towards the center. In the late subacute phase (2-4 weeks) the RBCs lyse and MetHb is released from the intracellular into the extracellular space. In this stage the edema gradually dissolves. Later on, in the chronic phase ( $>4$ weeks) the hematoma 

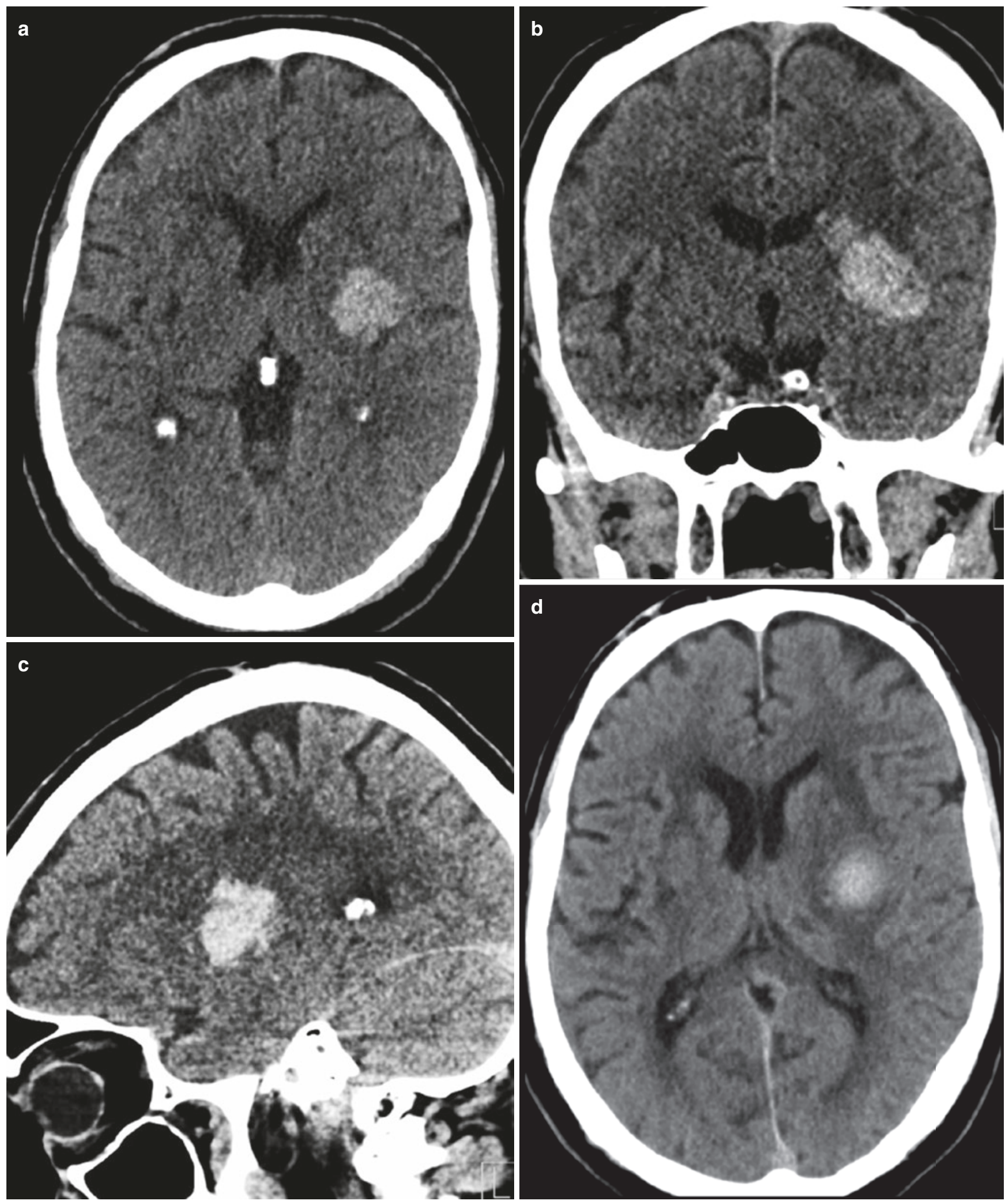

Fig. 5.1 Hypertensive hematoma. Axial (a) and reformatted coronal (b), and sagittal (c) CT images showing an acute hypertensive hematoma at the basal ganglia (putamen) dissecting into the corona radiata. The 10 day follow up CT images (d) shows the expected peripheral

hyperattenuation decrease and the presence of edema. The late follow up (1 year) CT image (e) shows the hematoma scar as a small area of calcification with peripheral hypoattenuation 


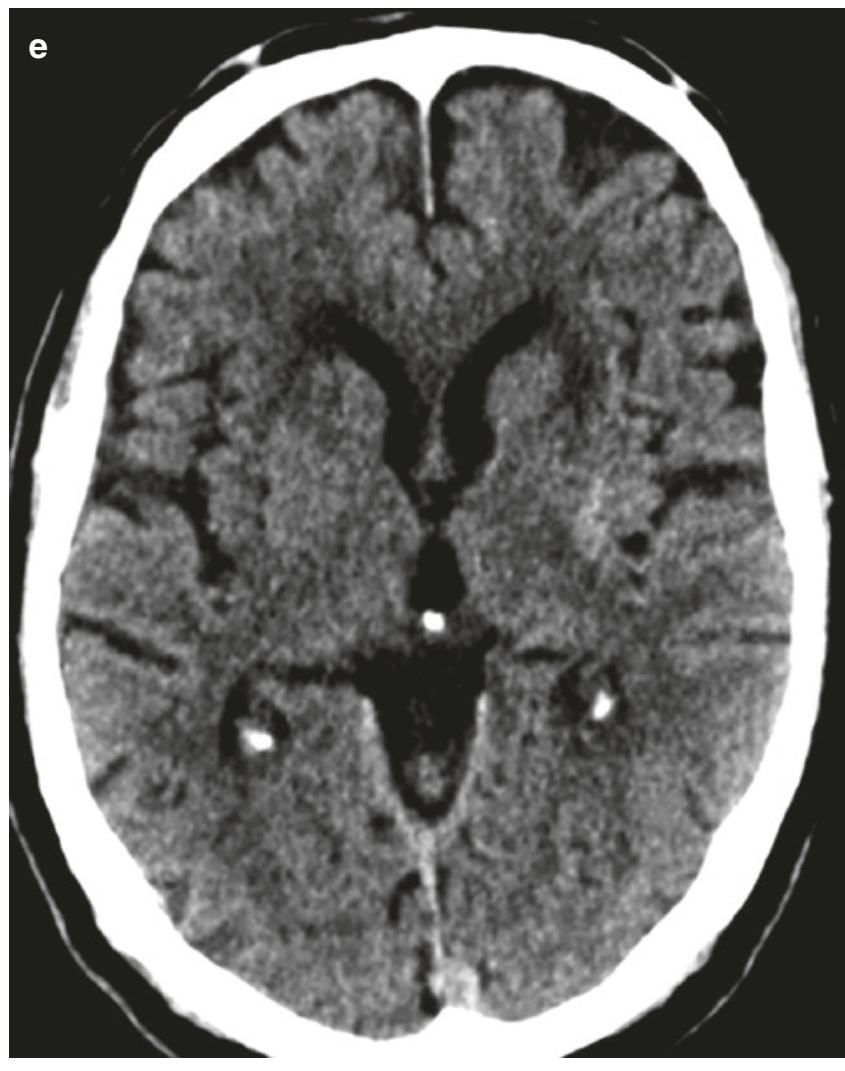

Fig. 5.1 (continued)
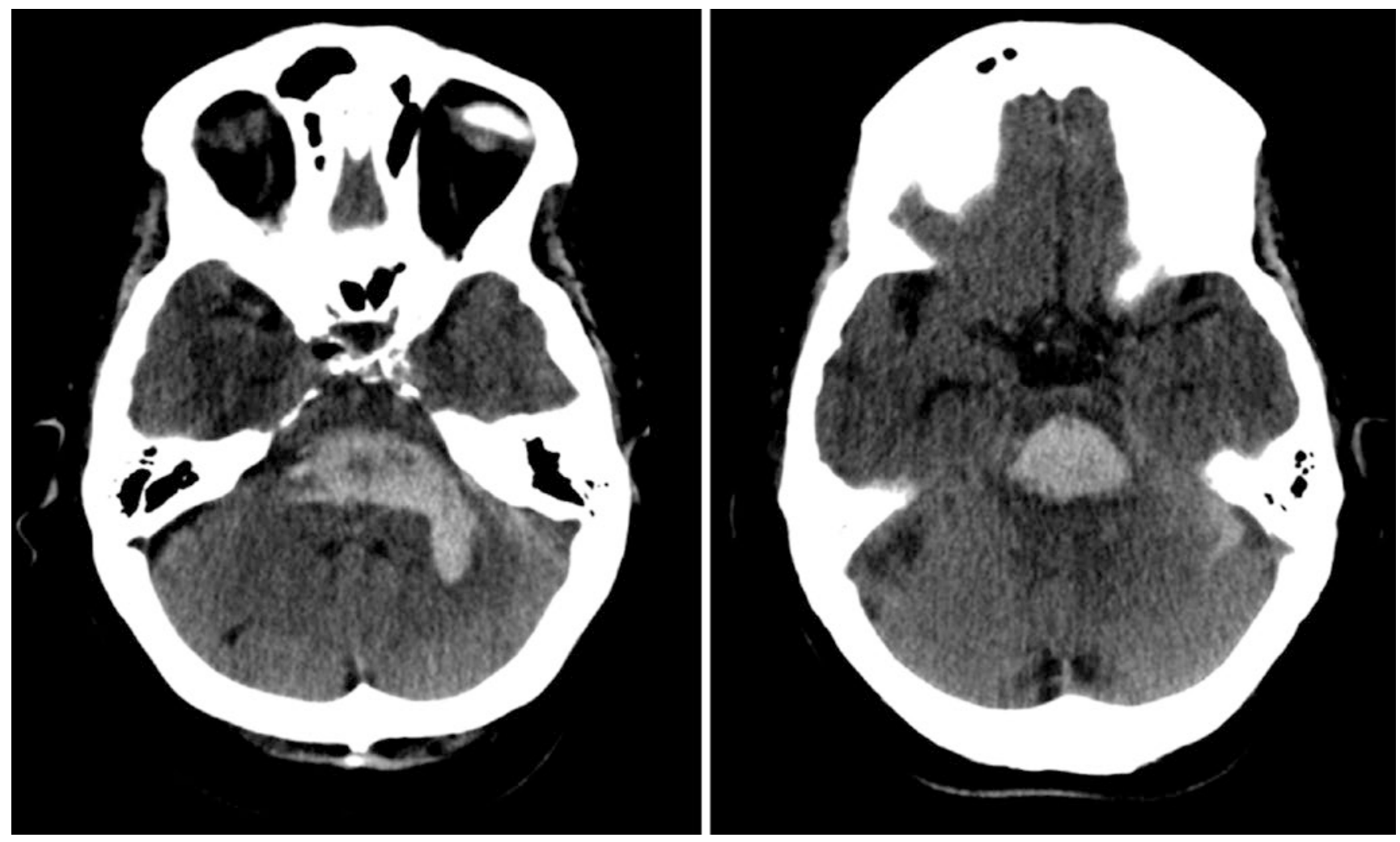

Fig. 5.2 Hypertensive hematoma. Axial CT images showing an acute (hyperattenuating) pontine hypertensive hematoma dissecting into the middle cerebellar peduncle and left medulary center 
with brain atrophy with sulcal and/or ventricular enlargement, and may exhibit focal calcification and peripheral gliosis.

Microhemorrhages are chronic small punctate brain hemorrhages only depicted by MRI using T2*W susceptibility imaging. They are depicted as small $(<5 \mathrm{~mm})$ rounded low $\mathrm{T} 2 *$ lesions which result from presumed ruptures of tiny vessels with diameters smaller than $200 \mu \mathrm{m}$. They are commonly associated with sporadic small vessel disease (systemic hypertension) and CAA, but can also be found in patients with nonsporadic small vessel disease, such as CADASIL and vasculitis, among others. They are also seen in ageing with increasing prevalence with older age and hypertension. The differential diagnosis of microbleeds includes non-hemorrhagic subcortical mineralization areas; pneumocephalus and hemorrhagic lesions such as cavernomas, hemorrhagic diffuse axonal injury, and microhemorrhagic brain metastasis.

The microhemorrhage pattern associated with chronic hypertension has a central distribution, located at the deep gray structures (basal ganglia and thalamus), cerebellum, and less importantly at the cerebral cortico-subcortical junction (Fig. 5.3). The CCA pattern has a peripheral distribution at the cerebral lobar cortico-subcortical areas with a posterior predominance (Fig. 5.4).

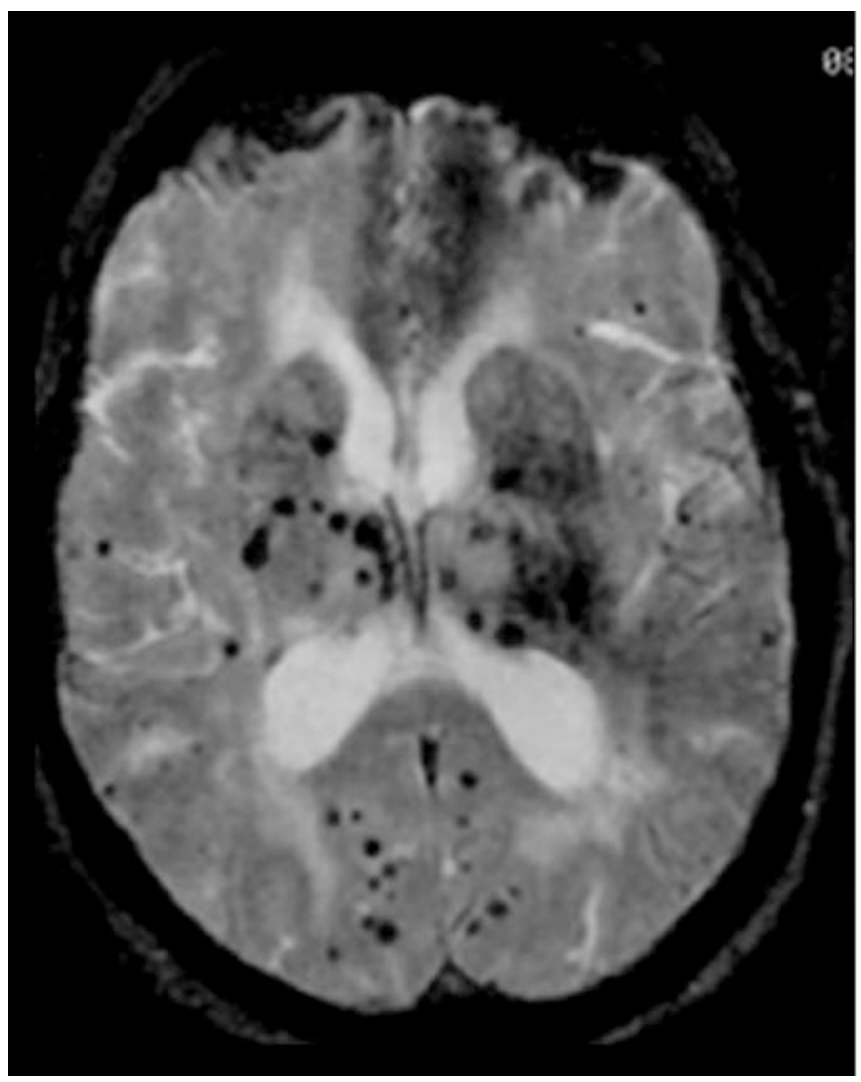

Fig. 5.3 Hypertensive microhemorrhages. Axial T2*-weighted MR images demonstrating the central pattern of microbleeds (small punctuate low intensity signal lesions) distribution, with thalamic, lenticular,
Subarachnoid and intraventricular hemorrhage manifest with hemorrhage into the CSF spaces presenting with increased attenuation on CT images (blood). The hemorrhage attenuation and MRI signal intensity follow the abovementioned evolution.

\section{Key Points}

- CT is the method of choice to detect acute intracranial hemorrhage

- CT and CTA allow the detection of active intracranial bleeding

- In the subacute stage MRI is superior to CT in the detection of subarachnoid hemorrhage

CT is the gold standard imaging method to depict acute SAH $[6,7]$. The sensitivity of CT to depict SAH during the first $6-12 \mathrm{~h}$ is $98-100 \%[6,7]$. The same diagnostic accuracy may be assumed for intraventricular hemorrhage. Over days, especially after the fifth day, the accuracy of CT decreases, and in the subacute phase a lumbar puncture may be needed to make the diagnosis [6].

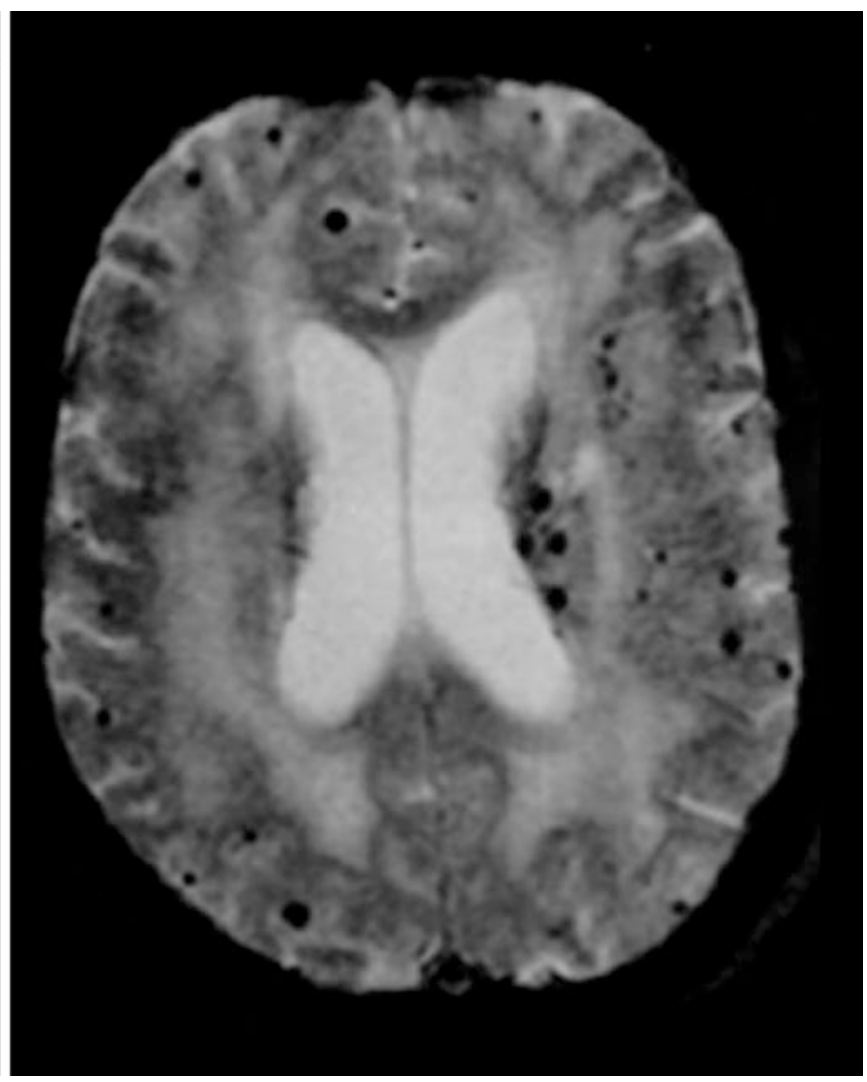

caudade, capsular and corona radiata locations, in association with a peripheral distribution at the lobar cortical-subcortical junction 

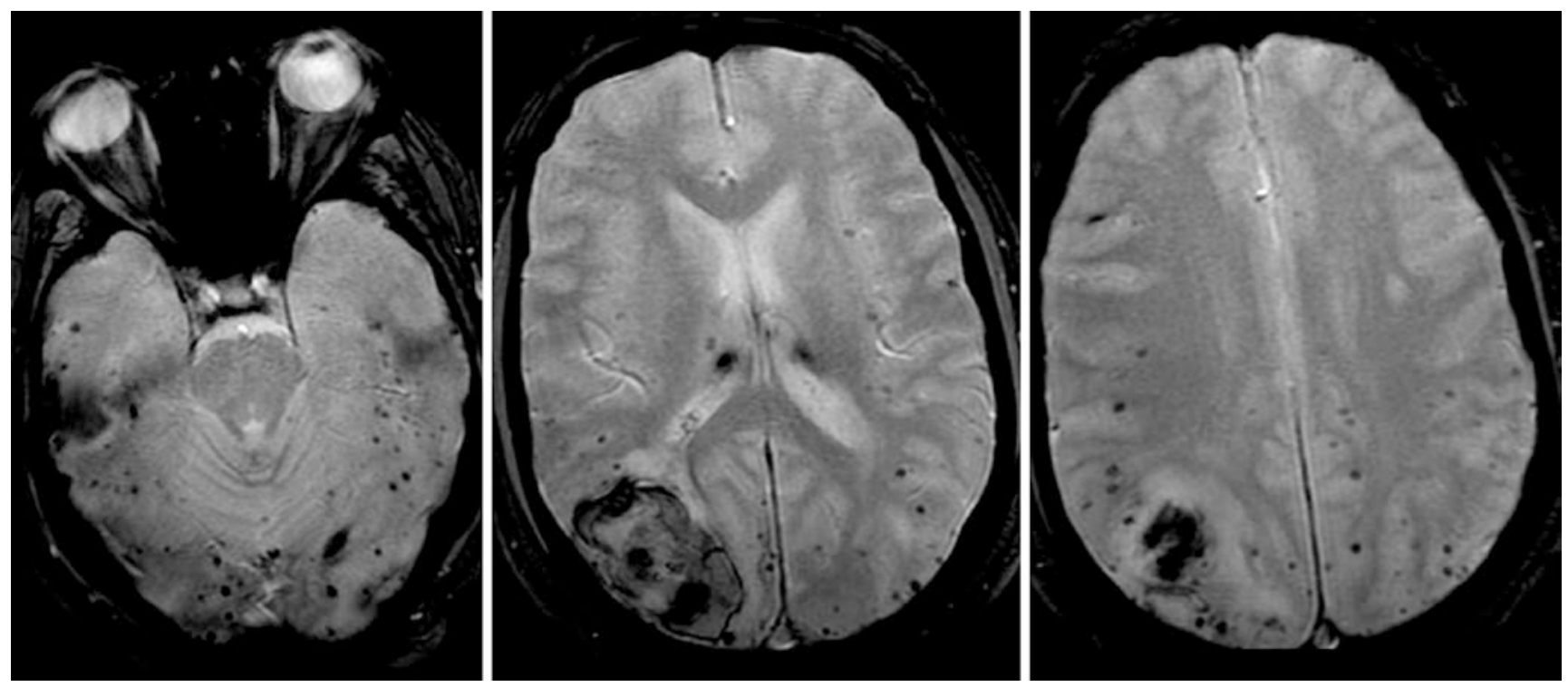

Fig. 5.4 Cerebral amyloid angiopathy (CAA) microhemorrhages. Axial T2-weighted MR images demonstrating the isolated peripheral pattern of microbleeds (small punctuate low intensity signal lesions)

Table 5.3 Modified Fisher grade

Grade 1 Minimal or diffuse thin SAH without IVH

Low risk for symptomatic vasospasm

Grade 2 Minimal or thin SAH with IVH

Grade 3 Thick cisternal clot without IVH

Grade 4 Cisternal clot with IVH

Intermediate risk for symptomatic vasospasm

High risk for symptomatic vasospasm

Recently, it has been shown that MRI has a very high accuracy, similar to $\mathrm{CT}$, to depict SAH in the acute phase, using FLAIR/DF sequences, and a higher accuracy than CT in the subacute and chronic phases (using FLAIR/DF and susceptibility weighted sequences) $[8,9]$. MRI is especially sensitive to chronic SAH, depicting the presence of superficial cortical hemosiderosis. This is an important diagnostic sign since its presence can help to suspect prior subarachnoid hemorrhage, or to substantiate the differential diagnosis of amyloid angiopathy.

The severity of the SAH is measured by the (modified) Fisher grade (Table 5.3). The pattern of blood distribution, namely basal cistern, superficial, or perimesencephalic distribution, allows the prediction of the etiology.

\subsection{Intracranial Hemorrhage Etiology}

There are multiple potential causes of hemorrhagic stroke, which can be primarily categorized according to the hemorrhage location and patient age, facilitating the imaging differential diagnosis approach, as summarized in Table 5.1. distribution with distribution at the lobar cortical-subcortical junction with posterior cerebral predominance, in association to a right occipital lobar hematoma

Additional imaging to evaluate an intracranial hemorrhage includes $\mathrm{CT}$ angiography to look for underlying vascular lesions and MRI to rule out other etiologies of ICH.

Vascular imaging is indicated whenever a vascular lesion is suspected, such as arterial aneurysms, pial arteriovenous malformations and dural arteriovenous fistula, or vasculitis. $\mathrm{CT}$ or MR venograms are very helpful to depict the presence of cerebral venous thrombosis, especially dural sinus thrombosis. CTA and MRA are the initial imaging methods. DSA is reserved for inconclusive cases, and for the treatment of some of the vascular lesions. The spot sign on CTA, reflecting contrast extravasation, depicted as an enhancing focus within the acute hematoma, deserves special attention since is associated with a higher risk of growth of the hematoma [10-14].

Brain hemorrhage accounts for up to $15 \%$ of all strokes [15]. Among the adult population brain hemorrhage is most commonly associated with systemic hypertension (Figs. 5.1, 5.2, and 5.3), followed by cerebral amyloid angiopathy (CAA) (Figs. 5.4 and 5.5). In younger patients and in children, vascular malformations are an important cause of brain hemorrhage. Cerebral venous thrombosis is also an important cause of brain hemorrhage in the young patient especially in females and during the pregnancy and puerperium. Hemorrhagic transformation of acute ischemic stroke and hemorrhagic brain tumors are also important causes of brain hemorrhage. Other potential causes of brain hemorrhage, representing $<10 \%$ of cases, include reversible cerebral vasoconstriction syndrome (RVCS), posterior reversible encephalopathy syndrome (PRES), vasculitis, coagulopathy disorders, drugs consumption (sympathomimetics, cocaine, amphetamine, ecstasy, among others), moyamoya disease, infections, and pre-eclampsia/eclampsia, among others. 

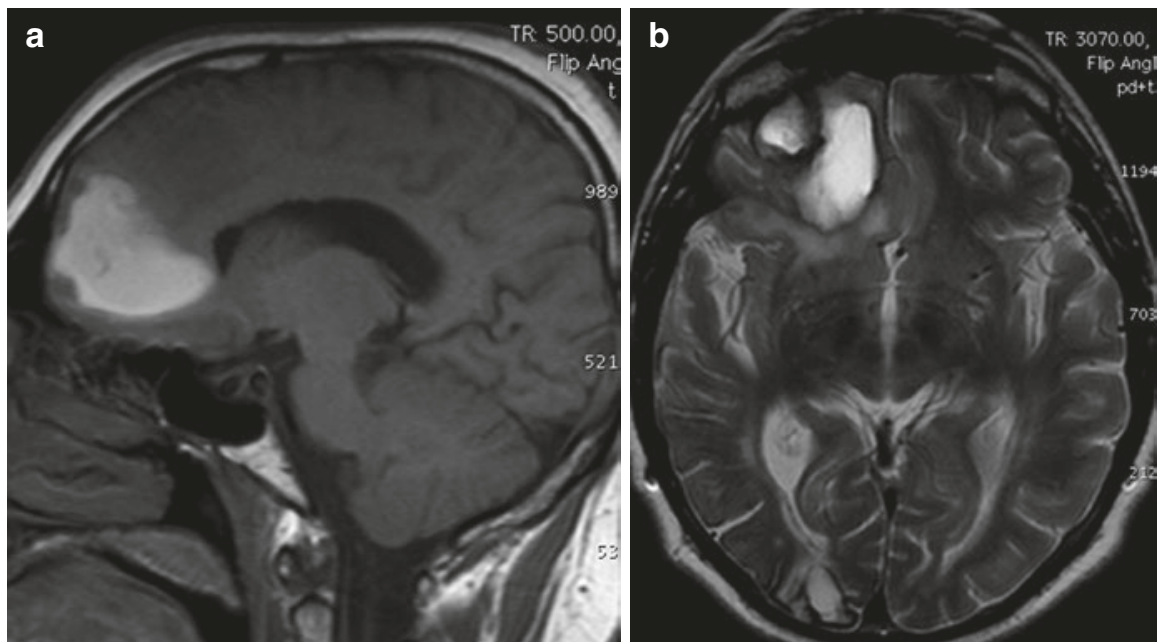

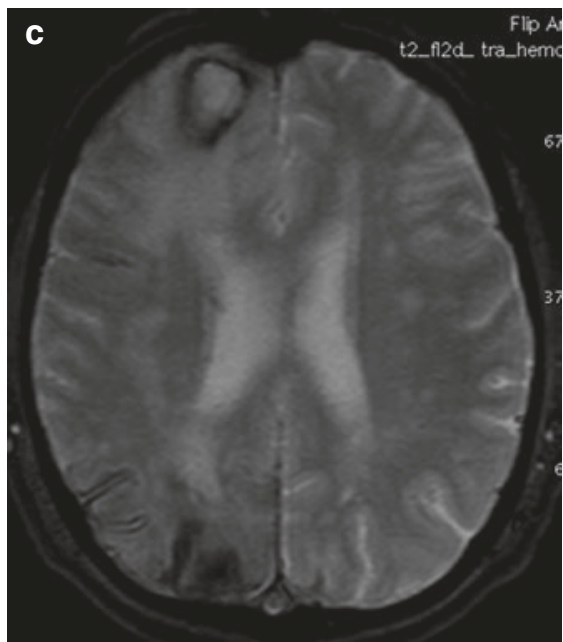

Fig. 5.5 Cerebral amyloid angiopathy (CAA). Axial T1-weighted MR image (a) depicting a frontal lobar hematoma in the subacute stage (extracellular methemoglobin). Axial T2-weight (b) and T2*-weighted

Hypertensive hemorrhage is the leading cause of cerebral hematomas in the adult population, being secondary to a long-standing hypertensive vasculopathy characterized by lipohyalinosis of small-to-medium penetrating arteries. Hematomas result from the rupture of small arterioles and have a rebleeding rate of up to $2 \%$ per year [16]. Typical locations for hypertensive hemorrhage (Figs. 5.1 and 5.2) include basal ganglia (in $62-65 \%$ of cases), thalamus (in $20-26 \%$ of cases), pons (in 5-10\% of cases), cerebellum (in approximately $6 \%$ of cases), and subcortical-cerebral lobar (in 1-2\%) of cases $[17,18]$. The association of leucoaraiosis, lacunar infarcts, and microbleeds with a central distribution pattern supports the diagnosis.

Cerebral amyloid angiopathy (CAA) is the second most common cause of spontaneous intracranial hemorrhage in adults and results from the vessel wall deposition of amyloid-b peptide in small leptomeningeal and cortical vessels. CAA is responsible for up to $50 \%$ of spontaneous hemorrhages in older patients ( $>65$ years) and its incidence increases with patient age, being present in up to one third of brain autopsies in patients above 60 years old, which reflects that in an ageing population it will be a very important cause of hemorrhage. The most common type of CAA is the sporadic type occurring in the elderly, but there are hereditary types that may present earlier, such as the HCHWA-Dutch type and HCHWA-Icelandic type with onset in the third/forth decade, and other reported familiar types. Imaging is an important criterion for the diagnosis of CAA according to the Boston diagnostic criteria (Table 5.4) [19]. CAA should be considered in cases of older patients with multiple lobar, cortical, and/or subcortical hemorrhages; superficial/cortical subarachnoid hemorrhage (superficial siderosis); and peripheral distributed microhemorrhages. Hemorrhages rarely involve the cerebellum and (c) images demonstrate additional cortical (superficial) subarachnoid hemorrhage at the right brain convexity and a chronic occipital lobar hematoma

Table 5.4 The modified Boston criteria for cerebral amyloid angiopathy (CAA)

\begin{tabular}{|c|c|c|}
\hline $\begin{array}{l}\text { Definite } \\
\text { CAA }\end{array}$ & $\begin{array}{l}\text { Full post-mortem } \\
\text { examination }\end{array}$ & $\begin{array}{l}\text { - Lobar, cortical, or cortical/ } \\
\text { subcortical hemorrhage } \\
\text { - Pathological evidence of } \\
\text { severe cerebral amyloid } \\
\text { angiopathy } \\
\text { - Absence of other diagnostic } \\
\text { lesion }\end{array}$ \\
\hline $\begin{array}{l}\text { Probable } \\
\text { CAA with } \\
\text { supporting } \\
\text { pathology }\end{array}$ & $\begin{array}{l}\text { Clinical data and } \\
\text { pathological tissue } \\
\text { (evacuated } \\
\text { hematoma or cortical } \\
\text { biopsy specimen) }\end{array}$ & $\begin{array}{l}\text { - Lobar, cortical, or } \\
\text { corticosubcortical hemorrhage } \\
\text { - Some degree of CAA in } \\
\text { specimen } \\
\text { - Absence of other diagnostic } \\
\text { lesion }\end{array}$ \\
\hline \multirow[t]{2}{*}{$\begin{array}{l}\text { Probable } \\
\text { CAA }\end{array}$} & Clinical data & $\begin{array}{l}\text { - Patient } 55 \text { years or older } \\
\text { - Absence of other diagnostic } \\
\text { lesion } \\
\text { - Absence of other cause of } \\
\text { hemorrhage or superficial } \\
\text { siderosis }\end{array}$ \\
\hline & $\begin{array}{l}\text { MRI or CT } \\
\text { demonstrating }\end{array}$ & $\begin{array}{l}\text { - Multiple hemorrhages } \\
\text { restricted to lobar, cortical, or } \\
\text { corticosubcortical regions } \\
\text { (cerebellar hemorrhages } \\
\text { allowed) of varying sizes/ } \\
\text { agesOr } \\
\text { - Single lobar, cortical, or } \\
\text { corticosubcortical hemorrhage } \\
\text { and focal (three or less sulci) } \\
\text { or disseminated (more than } \\
\text { three sulci) cortical superficial } \\
\text { siderosis }\end{array}$ \\
\hline \multirow[t]{2}{*}{$\begin{array}{l}\text { Possible } \\
\text { CAA }\end{array}$} & Clinical data & $\begin{array}{l}\text { - Patient } 55 \text { years or older } \\
\text { - Absence of other cause of } \\
\text { hemorrhage or superficial } \\
\text { siderosis }\end{array}$ \\
\hline & $\begin{array}{l}\text { MRI or CT } \\
\text { demonstrating }\end{array}$ & $\begin{array}{l}\text { - Single lobar, cortical, or } \\
\text { corticosubcortical } \\
\text { hemorrhageOr } \\
\text { - Focal or disseminated } \\
\text { superficial siderosis }\end{array}$ \\
\hline
\end{tabular}


the microbleeds spare the basal ganglia and brain stem (Fig. 5.4) [19].

\section{Key Points}

- CAA shows a different lesion pattern than hypertensive hemorrhage pattern and can be recognized by the presence of superficial siderosis

- In intracerebral hematomas early hematoma expansion, intracranial hypertension, brain herniation, and the presence of hydrocephalus need to be recognized early to allow for specific treatment

For brain hematomas, there are some red-flag imaging findings that should prompt our special attention. These cases may require early surgical treatment. It is essential to depict early hematoma expansion, intracranial hypertension and/or signs of mass effect, such as brain herniation, and the presence of hydrocephalus.

Predictors for worst outcome include hemorrhage volume, infratentorial location (brain stem and/or cerebellum), presence of intraventricular hemorrhage, and/or hydrocephalus $[15,20]$.

Hematoma volume can be measured from multiplanar reconstruction as the ratio $\mathrm{ABC} / 2$, being $\mathrm{ABC}$ the three orthogonal diameters (the diameters are multipliedlength $\times$ width $\times$ height—and divided by 2). It is also essential to depict intraventricular extension, since it represents a higher risk for hydrocephalus, especially if the clot is inside the aqueduct and/or IV ventricle. Brain herniation is associated with a dismal outcome, and may necessitate surgical decompression.

Other unfavorable factors of brain hematomas include advanced age, and high scores on the Glasgow Coma Score (GCS) and National Institutes of Health Stroke Scale (NHIHSS) at admission.

The ICH score allows an early estimation of the patient prognosis [20]. Higher scores are associated with higher 30 days mortality rates, namely of $13 \%, 26 \%, 72 \%$, and $97 \%$ corresponding to scores of $1,2,3$, and 4 , respectively (Table 5.5) [20]. Another brain hemorrhage prognostic score,

Table 5.5 ICH score

\begin{tabular}{|l|l|l|}
\hline ICH Score & & Points \\
\hline GCS score & $3-4$ & 2 \\
& $5-12$ & 1 \\
& $13-15$ & 0 \\
ICH volume $\left(\mathrm{cm}^{3}\right)$ & $\geq 30$ & 1 \\
& $<30$ & 0 \\
Intraventricular hemorrhage & Yes & 1 \\
& No & 0 \\
Infratentorial origin & Yes & 1 \\
\hline \multirow{2}{*}{ Age } & No & 0 \\
Total & $\geq 80$ & 1 \\
\hline & $<80$ & 0 \\
\hline & & $0-6$
\end{tabular}

that estimates the functional independence at 90 days, is the Functional Outcome in Primary Intracerebral Hemorrhage (FUNC) score [21].

It is also worthwhile to stress that up to $30 \%$ of brain hematomas will increase in size during the first $24 \mathrm{~h}$, especially during the first $3 \mathrm{~h}$ after presentation, which may be associated with neurological decline [22]. Repeat CT scans at $24 \mathrm{~h}$ or whenever there is a neurological decline is recommended [22].

Intracranial venous thrombosis (IVT) is an important cause of intracranial hemorrhage, especially in young patients and children. There is also a higher incidence during pregnancy and puerperium and in females under oral contraception. IVT results from the formation and propagation of a venous clot leading to the occlusion of the intracranial venous system components. Venous thrombosis features a wide spectrum of clinical and imaging presentation. Headache is the most common symptom. The diagnosis of IVT includes the demonstration of the thrombus/venous occlusion (direct signs), depicted by CT (which depicts only one third of cases as hyperdense thrombus inside the dural sinus—dense triangle sign-or cortical vein-cord sign-) or by MRI by the loss of the normal venous flow void by a clot that follows the same signal intensity progression previously described for parenchymal hematomas. MRV or CTV is also helpful to establish the diagnosis. It is also important to depict the presence of intracranial lesions (indirect signs), such as brain swelling, brain edema, venous infarction, intracranial hemorrhage, which are better defined by MRI [23].

Vascular malformations are the leading cause of spontaneous intracranial hemorrhage in children and young patients, including cavernomas and brain arteriovenous malformations (AVMs).

Brain AVMs are composed by abnormal arteriovenous shunts, occurring in a central nidus, which is the central area towards multiple feeding arteries converge and from which (early and enlarged) draining veins start. Most of the cases are sporadic, but they may be associated with HHT/ROW and RASA 1 mutation or being a part of metameric syndromes. Brain AVMs appear as a tangled cluster of abnormal vessels, dilated arteries and veins, which can be sulcal, parenchymal, and/or intraventricular. Brain AVMs are constituted by feeding arteries, with the nidus corresponding to the shunting area and veins that drain the malformation. The vessels present slightly hyperdense on $\mathrm{CT}$ and with flow voids on MRI. Small AVMs may only be depicted by DSA which is the gold standard imaging method. They may be associated with surrounding brain changes such as edema, gliosis, previous hemorrhage, and/or mass effect. The most common brain AVM classification used is the MartinSpetzler classification evaluating the size, location, and venous drainage of the brain AVM predicting the surgical risk for treatment. The hemorrhagic risk is higher for those AVM that have previously bled and for those with infratentorial, deep location, and deep venous drainage. 
Cavernomas are venous vascular malformations that are seen on CT as isodense or hyperdense lesions with calcifications in up to $40-60 \%$ of cases. On MRI their appearance is variable. Most commonly they have a central core with mixed signal from blood in different stages ("popcorn like"), being hyperintense on T1WI and hyper-hypointense on T2 WI. There is generally a completely $\mathrm{T} 2$ hypointense rim of hemosiderin and the contrast-enhancement is variable. The $\mathrm{T} 2 *$ /susceptibility sequences are the most sensitive imaging methods to depict cavernomas (Fig. 5.6). Cavernomas may appear only as small punctate lesions (more common in multiple cavernomas) that are poorly or not seen on $\mathrm{T} 1$ and $\mathrm{T} 2$ and only demonstrated on $\mathrm{T} 2 * /$ susceptibility sequences.
DSA is negative for the detection of cavernomas. Most of the cavernomas are sporadic but there are some familiar forms, such as CCM 1-3, for which cases multiple cavernomas are more common and an entire evaluation of the neuraxis is suggested. The most common presentation of cavernomas and brain AVM are hemorrhage, seizures, and neurological deficits.

Dural arteriovenous fistulae (AVF) tend to present in older patients. They are acquired vascular malformations in which there are abnormal connections between arteries that normally feed the meninges, bone, or muscles, but not the brain, and the dural sinuses or small venules within the dura mater. They are difficult to depict since there is no evident

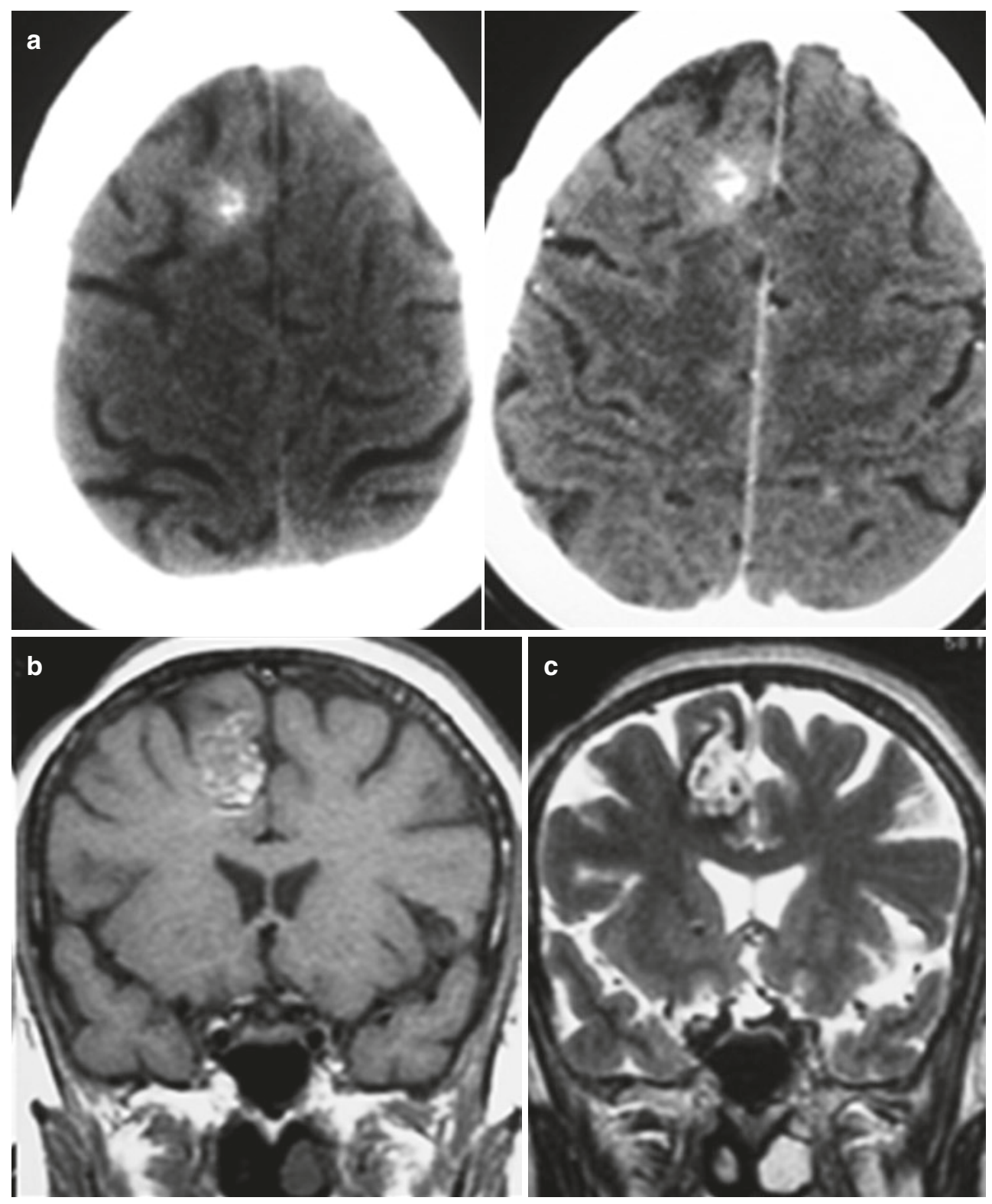

Fig. 5.6 Cavernoma. Axial CT images (a) showing a slight hyperattenuating superior frontal gyrus lesion with central calcifications. The coronal T1-weighted (b) and T2-weighted (c) MR images demonstrate the "popcorn-like" appearance with intralesional hyper- and hypoin- tense components in both sequences corresponding to blood in different stages. The axial T2*-weighted images (d) confirms the hemorrhagic nature of the lesion demonstrating the predominance of low signal with blooming effect 

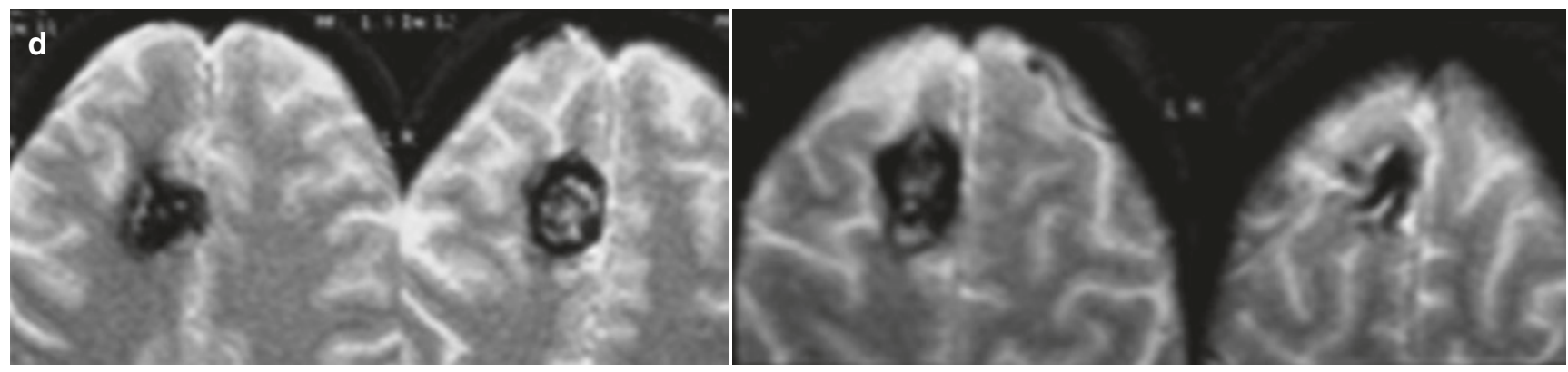

Fig. 5.6 (continued)

nidus and the feeding arteries are not as dilated as in brain AVM. The dilatation of meningeal arteries, presence of small arteries at the dura sinus and/or arterialized dural sinus, diffuse venous congestion and/or dilated veins should raise the suspicion of dural AVF. The hemorrhage risk of dural AVF is higher when a cortical venous reflux is present. The most common classifications, the Cognard and the Borden classifications, address the type of venous drainage and predict the future risk for hemorrhage.

Brain tumors are responsible for approximately $10 \%$ of intracranial bleedings. Early diagnosis allows for specific treatment. The most common causes of hemorrhage associated with brain tumors are intratumoral and/or extratumoral hemorrhage from the tumoral mass, generally in hypervascularized tumors. Other causes of hemorrhagic stroke may exist in neoplastic patients, such as malignancy induced coagulopathy, side effects from chemotherapy and/or radiotherapy, hemorrhagic infarcts resulting from thrombotic coagulopathy, arterial tumoral emboli (in association with heart myxoma), or from venous thrombosis in thrombotic coagulopathy or in association with venous compression/ invasion/thrombosis.

The tumors more commonly associated with brain hemorrhage are metastases, especially from breast and lung (bronchogenic) carcinoma, melanoma, choriocarcinoma, renal cell carcinoma, thyroid carcinoma, and primary highly vascularized tumors, such as high-grade gliomas.

There are some imaging findings that should raise the suspicion of the presence of an underlying tumor, such as atypical hematoma location; the presence of multiple hemorrhagic foci within the lesion; heterogeneous hematoma with different hemorrhage stages not following the usual chronological evolution or having a delayed evolution (due to hypoxic environment); the presence of fluid-fluid levels, that may represent blood-blood levels, blood-cystic/necrotic levels; the presence of an incomplete hemosiderin rim; the presence of irregular margins; the presence of a disproportionately larger edema; and/or the presence of non-hemorrhagic enhancing areas (Fig. 5.7). In the follow-up studies the presence of non-hemorrhagic enhancing areas may be more con- spicuous and there is generally a persisting mass effect and edema that does not subside completely.

Congenital or acquired coagulation disorders are important causes of spontaneous brain hemorrhages, since the number of patients under anticoagulation/antiplatelet therapy has been increasing over the years. These hemorrhages can occur in any intracranial compartment, even simultaneously. The presence of large or multiple and synchronous (same age/stage bleedings at CT/MRI) hemorrhages, and/or the presence of fluid-fluid hemorrhagic levels should raise the suspicion of coagulation disorder (Fig. 5.8).

It is not common to have brain hemorrhages associated with intracranial infections. Some of the typical examples include hemorrhagic encephalitis, such as herpes encephalitis, dengue fever, Crimean hemorrhagic fever-Congo virus, Epstein-Barr virus, and chikungunya virus, among others. Hemorrhage may also be a presentation of infectious vasculitis, such as varicella-zoster vasculitis. Fungal infection with vessel wall invasion may cause hemorrhage, e.g. in cases of aspergillosis and mucormycosis. Actinomycosis, paragonimiasis, cerebral malaria (due to hemorrhagic infarcts) may also present with devastating hemorrhagic lesions. Other causes of hemorrhage include infective endocarditis with septic emboli, hemorrhage associated with sepsis-induced coagulopathy and with infection treatment as in toxoplasmosis. In cases of meningitis, cerebral venous thrombosis and hemorrhagic transformation of ischemic infarcts may also lead to brain hemorrhage.

\subsection{Subarachnoid Hemorrhage and Intraventricular Hemorrhage}

The overall incidence of SAH is in between 9 and 20 per 100,000 person-years, accounting for up to $5 \%$ of all stroke cases [24-26]. The most common cause of spontaneous subarachnoid hemorrhage (SAH) is the rupture of an intracranial aneurysm, accounting for up to $80-85 \%$ of cases [24-26]. Ruptured saccular aneurysms commonly present with SAH located in the basal cisterns. 

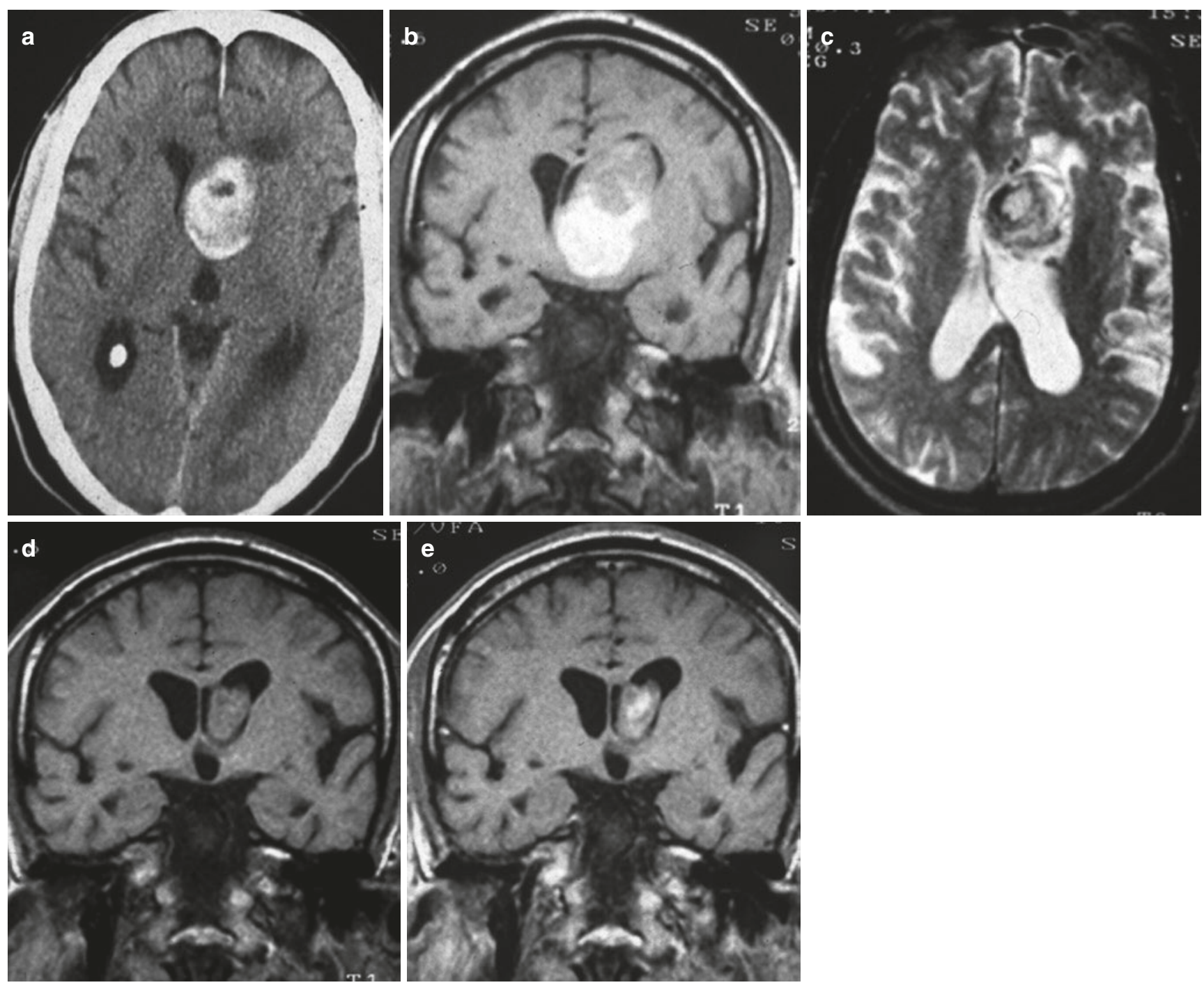

Fig. 5.7 Hemorrhagic tumor. Axial CT image (a) shows an acute hemorrhagic lesion (hyperattenuating) with intraventricular location (left frontal horn). The coronal T1-weighted (b) and axial T2-weighted (c) MR images demonstrate the heterogeneity of the signal intensities within the lesion not following the expected layered pattern of a pri- mary hemorrhage. The coronal T1-weighted (d) and coronal T1-weighted (e) post contrast images of follow up MRI performed after 3 weeks demonstrate the absorption of the hemorrhage and demonstrate the underlying intraventricular contrast-enhancing lesion corresponding to a high grade glioma
Additional causes of SAH include intracranial dissections, and for the cases with superficial/cortical subarachnoid hemorrhage, CAA, cortical venous thrombosis, vasculitis, reversible vasoconstriction syndrome (RVCS), distal arterial aneurysms, and dural arteriovenous fistulae (dAVF) should be considered.

Benign perimesencephalic SAH is a subgroup of SAH with an excellent prognosis. It accounts for up to $10 \%$ of cases and is believed to result from rupture of veins. Typically, the blood is collected at the perimesencephalic and prepontine cisterns and CTA and DSA are negative for the presence of aneurysm/vascular malformation.

\section{Key Points}

- DSA is the method of choice to screen for multiple aneurysms in all cases of SAH

- In cases of typical perimesencephalic SAH followup DSA studies are not required

Isolated intraventricular hemorrhage is an uncommon location for spontaneous hemorrhage. It generally results from the direct expansion of a parenchymal hematoma or the recirculation of blood in a SAH case. 

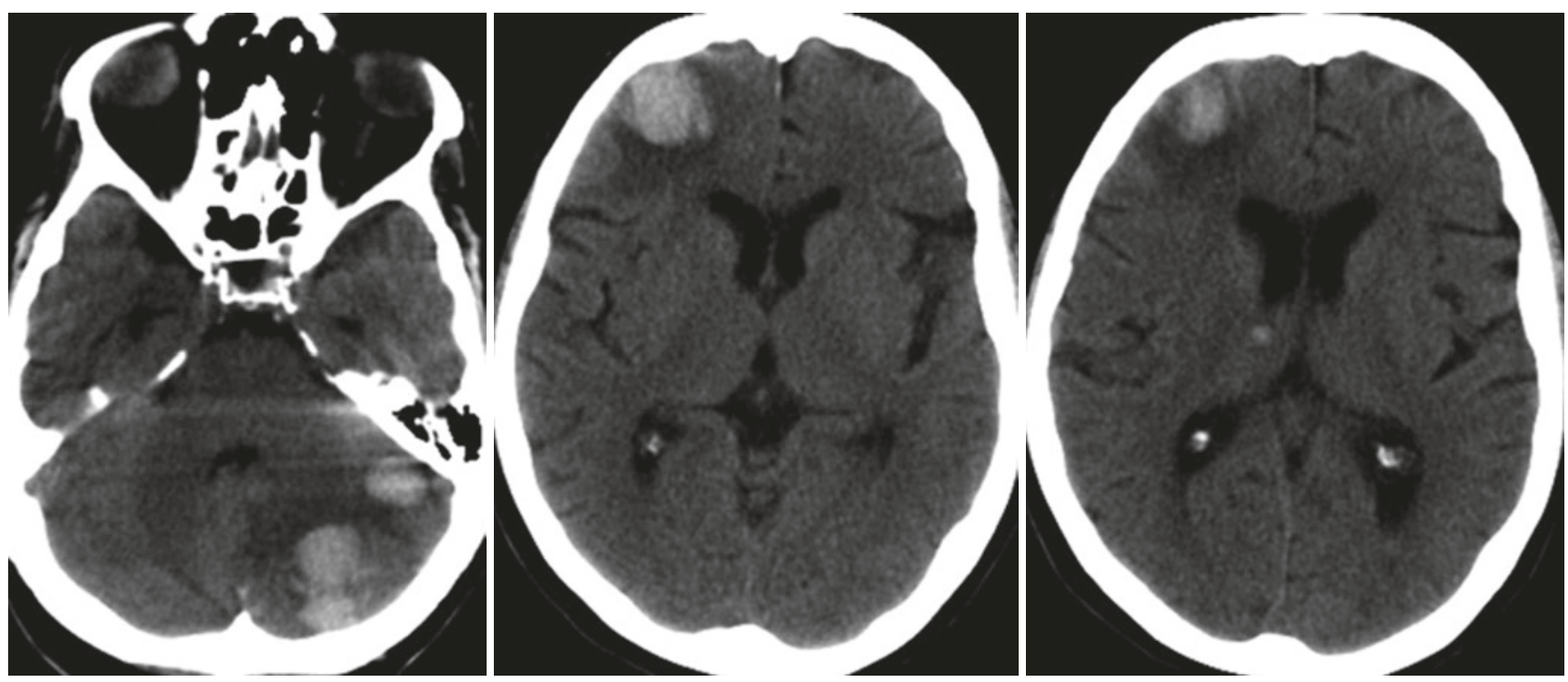

Fig. 5.8 Acquired coagulopathy associated brain hemorrhages. Axial CT images demonstrating multiple (left cerebellar, right inferior frontal and thalamic) acute hemorrhages with associated intraventricular hem- orrhage (blood level at the left atrium) in a patient with aplastic anemia and low platelets count $(12,000 / \mathrm{mcL})$

\subsection{Epidural/Subdural Hemorrhage}

Epidural and subdural hematomas are generally associated with head trauma. Exceptionally they may occur spontaneously in patients under anticoagulation or antiplatelet therapy. Isolated spontaneous EDH and SDH have been described most commonly in association with coagulopathies, dural and osteodural arteriovenous fistula, intracranial hypotension, and dural/bone tumors, especially metastases.

\subsection{Conclusions}

Imaging is essential for the management of intracranial hemorrhage. It allows for the depiction of the bleeding, the classification of the hemorrhage according to its size and location, to detect and/or exclude an underlying disorder, such as a vascular malformation, tumor, infection, among others. It also establishes the prognosis and guides the treatment.

\section{Take Home Messages}

- The imaging characteristics of intracranial hemorrhages vary over time for both CT and MRI

- In intracerebral hemorrhages the spot sign on CTA, reflecting contrast extravasation, deserves special attention since it is associated with a higher risk of growth of the hematoma
- CAA shows a different lesion pattern than hypertensive hemorrhage pattern and can be recognized by the presence of superficial siderosis

\section{References}

1. Feigin VL, et al. Global burden of stroke and risk factors in 188 countries, during 1990-2013: a systematic analysis for the Global Burden of Disease Study 2013. Lancet Neurol. 2016;15(9):913-24.

2. Broderick J, et al. Guidelines for the management of spontaneous intracerebral hemorrhage in adults: 2007 update: a guideline from the American Heart Association/American Stroke Association Stroke Council, High Blood Pressure Research Council, and the Quality of Care and Outcomes in Research Interdisciplinary Working Group. Circulation. 2007;116(16):e391-413. https://doi. org/10.1161/CIRCULATIONAHA.107.183689.

3. Parizel PM, et al. Intracranial hemorrhage: principles of CT and MRI interpretation. Eur Radiol. 2001;11(9):1770-83.

4. Venkatasubramanian $C$, et al. Natural history of perihematomal edema after intracerebral hemorrhage measured by serial magnetic resonance imaging. Stroke. 2011;42(1):73-80. https://doi. org/10.1161/STROKEAHA.110.590646.

5. Urday $\mathrm{S}$, et al. Targeting secondary injury in intracerebral haemorrhage - perihaematomal oedema. Nat Rev Neurol. 2015;11(2):11122. https://doi.org/10.1038/nrneurol.2014.264.

6. Bederson JB, et al. Guidelines for the management of aneurysmal subarachnoid hemorrhage: a statement for healthcare professionals from a special writing group of the Stroke Council, American Heart Association. Stroke. 2009;40(3):994-1025.

7. Perry JJ, et al. Sensitivity of computed tomography performed within six hours of onset of headache for diagnosis of subarachnoid haemorrhage: prospective cohort study. BMJ. 2011;343:d4277. 
8. Mitchell P, et al. Detection of subarachnoid haemorrhage with magnetic resonance imaging. J Neurol Neurosurg Psychiatry. 2001;70(2):205-11.

9. U-King-Im JM, et al. Current diagnostic approaches to subarachnoid haemorrhage. Eur Radiol. 2005;15(6):1135-47.

10. Safatli DA, et al. Predictors of 30-day mortality in patients with spontaneous primary intracerebral hemorrhage. Surg Neurol Int. 2016;7(Suppl 18):S510-7.

11. Kothari RU, et al. The ABCs of measuring intracerebral hemorrhage volumes. Stroke. 1996;27(8):1304-5.

12. Brouwers HB, et al. CT angiography spot sign in intracerebral hemorrhage predicts active bleeding during surgery. Neurology. 2014;83(10):883-9.

13. Delgado Almandoz JE, et al. The spot sign score in primary intracerebral hemorrhage identifies patients at highest risk of inhospital mortality and poor outcome among survivors. Stroke. 2010;41(1):54-60.

14. Fujii Y, et al. Multivariate analysis of predictors of hematoma enlargement in spontaneous intracerebral hemorrhage. Stroke. 1998;29(6):1160-6.

15. Broderick JP, et al. Intracerebral hemorrhage more than twice as common as subarachnoid hemorrhage. J Neurosurg. 1993;78(2):188-91.

16. Arakawa $S$, et al. Blood pressure control and recurrence of hypertensive brain hemorrhage. Stroke. 1998;29(9):1806-9.

17. Kranz PG, et al. Approach to imaging in patients with spontaneous intracranial hemorrhage. Neuroimaging Clin $\mathrm{N}$ Am. 2018;28(3):353-74.
18. Linn J, et al. Subarachnoid hemosiderosis and superficial cortical hemosiderosis in cerebral amyloid angiopathy. Am J Neuroradiol. 2008;29(1):184-6.

19. Greenberg SM, Charidimou A. Diagnosis of cerebral amyloid angiopathy: evolution of the Boston criteria. Stroke. 2018;49(2):491-7.

20. Hemphill JC 3rd, et al. The ICH score: a simple, reliable grading scale for intracerebral hemorrhage. Stroke. 2001;32(4): $891-7$.

21. Rost NS, et al. Prediction of functional outcome in patients with primary intracerebral hemorrhage: FUNC score. Stroke. 2008;39(8):2304-9. https://doi.org/10.1161/STROKEAHA.107. 512202 .

22. Brott T, et al. Early hemorrhage growth in patients with intracerebral hemorrhage. Stroke. 1997;28(1):1-5.

23. Vilela P. Imaging of cerebral venous and sinus thrombosis. In: Barkhof F, Jager R, Thurnher M, Rovira CA, editors. Clinical neuroradiology. Cham: Springer; 2019.

24. van Gijn J, Rinkel GJ. Subarachnoid haemorrhage: diagnosis, causes and management. Brain. 2001;124(2):249-78.

25. de Rooij NK, et al. Incidence of subarachnoid haemorrhage: a systematic review with emphasis on region, age, gender and time trends. J Neurol Neurosurg Psychiatry. 2007;78(12): 1365-72.

26. Zacharia BE, et al. Epidemiology of aneurysmal subarachnoid hemorrhage. Neurosurg Clin N Am. 2010;21(2):221-33.

Open Access This chapter is licensed under the terms of the Creative Commons Attribution 4.0 International License (http://creativecommons. $\mathrm{org} /$ licenses/by/4.0/), which permits use, sharing, adaptation, distribution and reproduction in any medium or format, as long as you give appropriate credit to the original author(s) and the source, provide a link to the Creative Commons license and indicate if changes were made.

The images or other third party material in this chapter are included in the chapter's Creative Commons license, unless indicated otherwise in a credit line to the material. If material is not included in the chapter's Creative Commons license and your intended use is not permitted by statutory regulation or exceeds the permitted use, you will need to obtain permission directly from the copyright holder. 